\title{
Testing relativity with high-energy astrophysical neutrinos
}

\author{
Jorge S. Díaz, ${ }^{1}$ V. Alan Kostelecký, ${ }^{1}$ and Matthew Mewes ${ }^{2}$ \\ ${ }^{1}$ Physics Department, Indiana University, Bloomington, Indiana 47405, USA \\ ${ }^{2}$ Physics Department, Swarthmore College, Swarthmore, Pennsylvania 19081, USA
}

(Received 12 September 2013; published 10 February 2014)

\begin{abstract}
The recent observation of high-energy astrophysical neutrinos can be used to constrain violations of Lorentz invariance emerging from a quantum theory of gravity. We perform threshold and Čerenkov analyses that improve existing bounds by factors ranging from about a million to $10^{20}$.
\end{abstract}

DOI: 10.1103/PhysRevD.89.043005

Astrophysical neutrinos offer exciting prospects for a distinctive perspective on the Universe, complementing existing photon and cosmic-ray techniques. In addition to enhancing studies of astrophysical objects, the detection of high-energy neutrinos from remote sources also provides opportunities for unique studies of physics at the fundamental level. In this work, we use the recent observations of TeV-PeV neutrinos in the IceCube detector at the South Pole and the evidence adduced in favor of their astrophysical origin $[1,2]$ to perform sensitive tests of Lorentz invariance, the foundational symmetry of relativity. Tiny observable violations of Lorentz invariance arising from new physics at the unification scale are proposed features of some underlying theories combining the fundamental interactions such as strings [3], but detecting these effects is expected to be challenging due to their likely suppression by factors involving the ratio of a light scale to the Planck scale. The advent of high-energy neutrino astrophysics vastly extends the prospects for tests with Planck-scale sensitivity because the combination of the long baseline and the high energy offers unique access to Plancksuppressed effects.

Neutrinos have several features making them well suited to studies of Lorentz invariance. The interferometric nature of neutrino oscillations makes them exquisitely sensitive to certain kinds of relativity violations, and several oscillation experiments have already achieved Planck-scale sensitivity [4]. In addition, the tiny or zero neutrino mass and weak neutrino interactions permit high sensitivity to types of Lorentz violation that are inaccessible to oscillation experiments $[5,6]$. For example, time-of-flight experiments comparing neutrino propagation against other particle species and studies of neutrino interactions and decay processes offer options for relativity tests that are unique and complementary to oscillation searches.

The general framework for studying Lorentz violation is the Standard-Model Extension (SME) [7], which is an effective field theory constructed from General Relativity and the Standard Model containing all operators for Lorentz violation. Each operator is controlled by a coefficient for Lorentz violation, and all operators describing
PACS numbers: 95.85.Ry, 11.30.Cp, 14.60.Lm, 98.70.Sa

neutrino propagation have recently been classified and enumerated [5]. For the purposes of this work, we can neglect oscillations of high-energy astrophysical neutrinos and can therefore work in the context of the general oscillation-free model or, where apposite, its isotropic limit. Also, since the observed high-energy IceCube events could be neutrinos or antineutrinos, focusing on CPT-even operators is appropriate. Here, we obtain constraints on oscillation-free coefficients for CPT-even Lorentz violation deduced from threshold effects in hadron decays and from Čerenkov radiation. Except where stated otherwise, significant Lorentz violation is assumed to be limited to the neutrino sector, compatible with the SME framework and current experimental bounds [8]. The constraints reported in this work improve existing limits by factors ranging from $10^{6}$ to $10^{20}$.

In the relativistic, oscillation-free, and CPT-even limit, the dispersion relation for a high-energy neutrino or antineutrino of energy $E$ and momentum $\boldsymbol{p}$ is [5]

$$
E(\boldsymbol{p})=|\boldsymbol{p}|-\sum_{d j m}|\boldsymbol{p}|^{d-3} Y_{j m}(\hat{\boldsymbol{p}})\left(c_{\mathrm{of}}^{(d)}\right)_{j m},
$$

where $d=4,6,8, \ldots$ is the mass dimension of the underlying operator in the field-theoretic action, $j, m$ are conventional angular-momentum indices with $0 \leq j \leq d-2$, and $\left(c_{\mathrm{of}}^{(d)}\right)_{j m}$ are oscillation-free coefficients for Lorentz violation. This expression omits the usual mass term, which decreases with energy and can be neglected at high energies, along with contributions from the odd- $d$ coefficients $\left(a_{\mathrm{of}}^{(d)}\right)_{j m}$ associated with CPT-odd operators in the SME, which appear with opposite signs for neutrinos and antineutrinos. The Lorentz-violating modifications in Eq. (1) introduce unconventional energy dependence as well as dependence on the propagation direction. For each operator dimension $d$, there are $(d-1)^{2}$ independent coefficients $\left(c_{\mathrm{of}}^{(d)}\right)_{j m}$, giving nine independent constant observables at the minimal dimension $d=4$ and 25 observables at the next order $d=6$. The setting of Eq. (1) in the broader model context is discussed in Ref. [5]. 
Commuting boost generators produces rotations, so every dispersion relation incorporating Lorentz violation is necessarily accompanied by some type of direction dependence. However, for situations where available data are insufficient to perform a complete analysis of coefficients for Lorentz violation at a given dimension $d$, it is sometimes convenient to work within the isotropic limit of the dispersion relation (1) to achieve order-of-magnitude estimates of the maximal potential sensitivity. In this isotropic limit, which holds only in a special frame, the dispersion relation (1) reduces to [5]

$$
E(\boldsymbol{p})=|\boldsymbol{p}|-\sum_{d}|\boldsymbol{p}|^{d-3 \stackrel{\circ}{c}(d)},
$$

where the isotropic coefficients $\dot{c}^{(d)}$ are defined by $\grave{c}^{(d)} \equiv\left(c_{\mathrm{of}}^{(d)}\right)_{00} / \sqrt{4 \pi}$. The special frame is often assumed to be the rest frame of the cosmic microwave background radiation, which differs from an Earth-based frame by a boost velocity $\simeq 10^{-3}$, implying that the isotropic dispersion relation (2) can provide only an approximation to an exact treatment of Lorentz violation involving astrophysical neutrinos. Another disadvantage of the isotropic approximation is that neutrino speeds exceeding light speed occur only for negative $i^{(d)}$, so analyses based on threshold effects or Čerenkov radiation can yield only lower bounds on the isotropic coefficients. In contrast, the direction dependence encoded in the full dispersion relation (1) permits two-sided bounds, given the availability of data with sufficient sky coverage.

While there is evidence that the IceCube $\mathrm{TeV}-\mathrm{PeV}$ neutrinos are astrophysical [1,2], an atmospheric origin for these events cannot yet definitively be excluded. We therefore begin by considering this more conservative scenario for the two PeV IceCube events [1].

Suppose first that muon neutrinos or antineutrinos are produced by atmospheric decays of hadrons $h$ via $h \rightarrow \mu+\nu_{\mu}$. In the presence of unconventional dispersion relations, these decays can display sharp threshold effects, including becoming forbidden or allowed according to the neutrino energy $[9,10]$. Conservation of energy implies a threshold on the energy defect $\delta E(\boldsymbol{p}) \equiv E(\boldsymbol{p})-|\boldsymbol{p}|$ given by $\delta E(\boldsymbol{p}) \leq \frac{1}{2} \Delta M^{2} /|\boldsymbol{p}|$, where $\Delta M=M_{h}-M_{\mu}$ is the difference between the hadron and muon masses [5]. Above this energy, production of atmospheric neutrinos cannot occur. The dispersion relation (1) shows this effect is generically direction dependent, but since only two PeV events are available we work in the isotropic limit (2) for which the threshold condition becomes

$$
-\sum_{d}|\boldsymbol{p}|^{d-2} \stackrel{\circ}{c}(d)^{2} \leq \frac{1}{2} \Delta M^{2}
$$

At lower energies, the atmospheric neutrino flux is dominated by the decays of $\pi$ and $K$ mesons, for which $\frac{1}{2} \Delta M^{2} \simeq 5.8 \times 10^{-4} \mathrm{GeV}^{2}$ and $\frac{1}{2} \Delta M^{2} \simeq 7.5 \times 10^{-2} \mathrm{GeV}^{2}$, respectively. However, at higher energies the prompt decays of short-lived charmed hadrons with $M \simeq 2 \mathrm{GeV}$ are expected to dominate, for which $\frac{1}{2} \Delta M^{2} \simeq 2 \mathrm{GeV}^{2}$. Taking one coefficient in Eq. (3) at a time, this value of $\Delta M^{2}$ and $|\boldsymbol{p}| \simeq 1 \mathrm{PeV}$ yields estimated one-sided constraints on the isotropic SME coefficients ${ }_{c}^{(d)}$ under the assumption of an atmospheric origin for the two PeV events. The results for dimensions $d \leq 10$ are compiled in the second column of Table I.

Comparable sensitivities can be obtained through limits on the energy loss due to Čerenkov-like decays of atmospheric neutrinos as they propagate to the detector. The Cerenkov radiation can occur when the maximum attainable neutrino speed exceeds that of the emitted particles, which for the Lorentz-invariant case is the speed of light [11]. Consider a superluminal neutrino of four-momentum $p=(E, \boldsymbol{p})$ that decays via the neutral-current process $\nu_{\mu} \rightarrow \nu_{\mu}+e^{+}+e^{-}$into a neutrino of momentum $p^{\prime}=\left(E^{\prime}, \boldsymbol{p}^{\prime}\right)$ and a charged-lepton pair with momenta $k=\left(k^{0}, \boldsymbol{k}\right)$ and $k^{\prime}=\left(k^{\prime 0}, \mathbf{k}^{\prime}\right)$. The rate of energy loss is given by an integral of the form [5]

$$
\frac{d E}{d x}=-\frac{C}{8} \int \frac{\kappa^{0} \boldsymbol{\kappa}^{\prime 2} \frac{\partial\left|\kappa^{\prime}\right|}{\partial \kappa_{0}}}{\left(\kappa^{2}-M_{Z}^{2}\right)^{2}} \frac{q \cdot k q^{\prime} \cdot k^{\prime}}{q_{0} k_{0} q_{0}^{\prime} k_{0}^{\prime}} d^{3} p^{\prime} d \Omega_{\kappa^{\prime}}
$$

where $C=2 G_{F}^{2}\left(1-4 \sin ^{2} \theta_{W}+8 \sin ^{4} \theta_{W}\right) M_{Z}^{4} /(2 \pi)^{5}$, the four-vectors $\kappa, \kappa^{\prime}$ are defined as $\kappa=k+k^{\prime}, \kappa^{\prime}=k-k^{\prime}$, and $q / q^{0}=(1, \hat{\boldsymbol{p}}), q^{\prime} / q^{\prime 0}=\left(1, \hat{\boldsymbol{p}}^{\prime}\right)$. The solid angle $d \Omega_{\kappa^{\prime}}$ is associated with the vector $\boldsymbol{\kappa}^{\prime}$, while the integral is restricted to the phase space for which $p=p^{\prime}+k+k^{\prime}$. A typical baseline distance for atmospheric neutrinos is on the order of $1000 \mathrm{~km}$. Neutrinos with significant superluminal speeds would dissipate much of their energy via Čerenkov pair production before being detected, so the observation of high-energy neutrinos implies a limit on the rate of energy loss. The characteristic propagation distance associated with this energy loss is given by the distortion distance $D(E)=-E /(d E / d x)$. Numerically performing the integral in Eq. (4), we can determine $D(E)$ for various fixed values of each coefficient ${ }^{\circ}(d)$ in turn. Large negative values of $i^{(d)}$ produce a distortion distance much smaller than

TABLE I. Estimated lower bounds on the isotropic coefficients $\stackrel{\circ}{c}(d)^{(d)}$ obtained using the two PeV IceCube events [1]. Units are $\mathrm{GeV}^{4-d}$.

\begin{tabular}{lccc}
\hline \hline Coefficient & $\begin{array}{c}\text { Atmospheric } \\
\text { threshold }\end{array}$ & $\begin{array}{c}\text { Atmospheric } \\
\text { Čerenkov }\end{array}$ & $\begin{array}{c}\text { Astrophysical } \\
\text { Čerenkov }\end{array}$ \\
\hline$\dot{c}^{(4)}$ & $>-2 \times 10^{-12}$ & $>-3 \times 10^{-13}$ & $>-5 \times 10^{-19}$ \\
$\dot{c}^{(6)}$ & $>-2 \times 10^{-24}$ & $>-3 \times 10^{-25}$ & $>-5 \times 10^{-31}$ \\
$\dot{c}^{(8)}$ & $>-2 \times 10^{-36}$ & $>-2 \times 10^{-37}$ & $>-5 \times 10^{-43}$ \\
$\dot{c}^{(10)}$ & $>-2 \times 10^{-48}$ & $>-2 \times 10^{-49}$ & $>-5 \times 10^{-55}$ \\
\hline \hline
\end{tabular}


$1000 \mathrm{~km}$, which would imply a substantial energy loss. Identifying the value of $i^{(d)}$ for which $D(E) \simeq 1000 \mathrm{~km}$ therefore gives an estimated lower constraint on $\dot{c}^{(d)}$. The third column of Table I lists the bounds obtained in this manner for $d=4,6,8,10$, assuming an atmospheric origin for the PeV IceCube events.

Significantly tighter constraints hold if the high-energy neutrinos detected by IceCube are of astrophysical origin, as has been suggested by several researchers $[1,2,12]$. The large propagation distance implies that even a minuscule $d E / d x$ has a substantial effect. Neutrinos above the threshold for Čerenkov-like decays lose energy until they are at or near threshold, so observed astrophysical neutrinos must have energies near or below threshold. For a positive energy defect $\delta E$, the threshold energy is

$$
\begin{aligned}
E(\boldsymbol{p}) & =\sqrt{\boldsymbol{k}^{2}+m_{e}^{2}}+\sqrt{\boldsymbol{k}^{\prime 2}+m_{e}^{2}}+E\left(\boldsymbol{p}^{\prime}\right) \\
& \geq \sqrt{\left(\boldsymbol{k}+\boldsymbol{k}^{\prime}\right)^{2}+4 m_{e}^{2}}+\sqrt{\boldsymbol{p}^{\prime 2}} \geq \sqrt{\boldsymbol{p}^{2}+4 m_{e}^{2}} .
\end{aligned}
$$

Squaring both sides of the above inequality and dropping the small $\delta E^{2}$ term produces the threshold condition $|\boldsymbol{p}| \delta E(\boldsymbol{p}) \approx 2 m_{e}^{2}$. The condition that observed neutrinos are near or below this threshold then yields

$$
-\sum_{d j m}|\boldsymbol{p}|^{d-2} Y_{j m}(\hat{\boldsymbol{p}})\left(c_{\mathrm{of}}^{(d)}\right)_{j m} \lesssim 2 m_{e}^{2} .
$$

In the isotropic limit and assuming an astrophysical origin, this inequality implies the two PeV IceCube events provide the lower bounds on $\dot{c}^{(d)}$ listed in the last column of Table I, where each coefficient is taken nonzero in turn. For $d=4$, these results are consistent with analyses of isotropic velocity defects [13]. For each listed value of $d$, the values obtained here sharpen by about a millionfold the existing constraints on isotropic coefficients for Lorentz violation in neutrinos, reaching for the first time levels competitive with other SME astrophysical constraints from photon and fermion dispersion relation [14].

The above analysis shows that the two PeV events lead to stringent one-sided limits on simple isotropic models involving only the coefficients $i^{(d)}$. However, the complete two-sided space of coefficients $\left(c_{\mathrm{of}}^{(d)}\right)_{j m}$ for each $d$ is accessible only with a larger number of events involving neutrinos with different propagation directions. For each observed event, the inequality (6) provides a one-sided bound on a linear combination of the coefficients $\left(c_{\mathrm{of}}^{(d)}\right)_{j m}$ fixed by the magnitude $|\boldsymbol{p}|$ and direction $\hat{\boldsymbol{p}}$ of the neutrino momentum, representing a boundary plane in the $(d-1)^{2}$ dimensional coefficient space. One can therefore expect that at least $(d-1)^{2}+1$ events are needed to extract twosided constraints on all the possible types of Lorentz violation allowed by operators of mass dimension $d$. In practice, the events must also be sufficiently well distributed across the sky to insure their linear independence.
Ideally, combining constraints from multiple neutrinos would enclose a small volume in the coefficient space containing the zero-coefficient Lorentz-invariant limit. Probing the allowed range of coefficients within this volume would then permit the identification of robust two-sided limits on individual coefficients for Lorentz violation. However, this ideal scenario is unattainable in practice because the direction-independent isotropic coefficient $i^{(d)}$ enters all linear combinations of the form (6) accompanied by a negative multiplier, and hence it has no upper bound. At best, the bounding surface opens in the positive $\dot{c}^{(d)}$ direction, so all the anisotropic coefficients are unconstrained as $\dot{c}^{(d)} \rightarrow \infty$. Individual two-sided bounds are therefore impossible in the absence of a two-sided bound on $i^{(d)}$. Instead, we determine here complete limits on anisotropic effects for each $j \neq 0$ and then comment on the prospects for isotropic bounds. We find that the 28 IceCube events at $\mathrm{TeV}-\mathrm{PeV}$ energies $[1,2]$ suffice in both number and sky distribution to place complete constraints at $d=4$ and $d=6$ for each $j$. Note that the existing data also permit partial coverage of cases with $d \geq 8$, but a complete treatment remains refractory until further events are accumulated.

Numerical bounds can be calculated using a modified simplex method of linear programming [15], which we briefly describe here in the context of the nine anisotropic coefficients with $d=4$ as an illustration. The procedure begins by writing the 28 individual bounds obtained from Eq. (6) in the form

$$
-\sum_{j m}\left[|\boldsymbol{p}|^{2} Y_{j m}(\hat{\boldsymbol{p}}) /\left(2 m_{e}^{2} s\right)\right]\left(s\left(c_{\mathrm{of}}^{(d)}\right)_{j m}\right)<1,
$$

where $s$ is a constant scaling factor chosen so that the terms in square brackets are of order one, thereby reducing precision error. The nine scaled coefficients $s\left(c_{\mathrm{of}}^{(d)}\right)_{j m}$ are placed in a column matrix $c$ with entries labeled $c_{n}$, $n=1,2, \ldots, 9$. The 28 constraints can then be written as the matrix equation $A \cdot c<B$, where $B$ is a 28-dimensional column matrix and $A$ is a $28 \times 9$ matrix of constants. We can account for the possibility of unbounded coefficients by augmenting the 28 constraint equations by the conditions $c_{n}<\infty$ and $-c_{n}<\infty$, where $\infty$ is the numerical infinity. This increases the row dimension of our matrix inequality to $28+9+9=46$, with the components of the matrix $B$ being either 1 or $\infty$. Adding these constraints allows the search method to step to the boundary at infinity, indicating an unbounded coefficient. The simplex technique then introduces 46 non-negative slack variables in a column matrix $S$ and writes the matrix inequality as $A \cdot c+S=B$. The procedure starts with the solution $c=0, S=B$ and then takes steps within the bounded region to maximize a given coefficient. The initial basic variables are the slack variables, and the initial free variables are the coefficients, so a tableau of the form 


\begin{tabular}{cccccccccc}
\hline & $c_{1}$ & $c_{2}$ & $\ldots$ & $c_{9}$ & $S_{1}$ & $S_{2}$ & $\ldots$ & $S_{46}$ & \\
\hline$S_{1}$ & $A_{1,1}$ & $A_{1,2}$ & $\ldots$ & $A_{1,9}$ & 1 & 0 & $\ldots$ & 0 & $B_{1}$ \\
$S_{2}$ & $A_{2,1}$ & $A_{2,2}$ & $\ldots$ & $A_{2,9}$ & 0 & 1 & $\ldots$ & 0 & $B_{2}$ \\
$\vdots$ & $\vdots$ & & & & & & & $\vdots$ & $\vdots$ \\
$S_{46}$ & $A_{46,1}$ & $A_{46,2}$ & $\ldots$ & $A_{46,9}$ & 0 & 0 & $\ldots$ & 1 & $B_{46}$ \\
\hline
\end{tabular}

characterizes the initial system. The first column lists the basic variables, while the last column tracks their values. The other columns are associated with the coefficients and the slack variables. A standard matrix pivot operation moves a zero free variable into the list of nonzero basic variables, displacing one of the original basic variables. The strategy behind maximizing a coefficient $c_{n}$ has two steps: first, make $c_{n}$ a basic variable by performing a pivot around the element in the $c_{n}$ column that gives the largest value of $c_{n}$ and, second, repeatedly try all pivots leaving $c_{n}$ as a basic variable, accepting those that increase $c_{n}$ and exiting when no allowed pivots increase $c_{n}$. In both steps, pivots that give negative slack variables are rejected. The minimum for the coefficient $c_{n}$ can be found by writing the matrix inequality as $(-A) \cdot(-c)<B$ and finding the maximum of $-c_{n}$, which in practice means that minima are obtained by changing the sign of the $A$ matrix and applying the above maximization procedure. Finally, the maxima and minima are divided by the scale factor $s$ to yield the desired bounds on the coefficient combinations.

Table II displays constraints on the SME coefficients $\left(c_{\mathrm{of}}^{(4)}\right)_{j m}$ and $\left(c_{\mathrm{of}}^{(6)}\right)_{j m}$ obtained using this simplex method applied to the IceCube data $[1,2]$ and reported in the Suncentered frame [16]. We adopt a cosmological origin for the IceCube events, but the order of magnitude of the constraints displayed holds even for a galactic origin. For the complex coefficients with $m \neq 0$, bounds on the real and imaginary parts are found separately. The first column lists the values of $d$ and $j$ used in the routine, while the third column contains the coefficient involved. The second and fourth columns provide the resulting numerical lower and upper bounds, respectively. For oscillation-free coefficients with $d=4$, the results in Table II represent improvements of well over a millionfold over existing constraints [8], while for $d=6$ the improvements are by factors up to about $10^{20}$.

An interesting open issue is the prospect for independent upper bounds on $i^{(d)}$. For $d=4$, the current best upper bound is provided by Altschul [17], who finds $\dot{c}^{(4)} \lesssim 10^{-11}$ is required to exclude the proton decay $p \rightarrow n+e^{+}+\nu$ in cosmic rays with energies $\simeq 10^{20} \mathrm{eV}$. Generalizing this analysis to arbitrary $d$ and neglecting possible Lorentz violation in protons, we obtain

$$
\dot{c}^{(d)} \lesssim \frac{m_{n}}{\left|\boldsymbol{p}_{p}\right|^{d-3}} \simeq 10^{33-11 d} \mathrm{GeV}^{4-d} .
$$

This yields $\stackrel{\circ}{c}^{(6)} \lesssim 10^{-33} \mathrm{GeV}^{-2}$ and offers good prospects for constraints on $i^{(d)}$ for $d \geq 8$. However, achieving a
TABLE II. Constraints on dimensionless coefficients $\left(c_{\mathrm{of}}^{(4)}\right)_{j m}$ and on $\left(c_{\text {of }}^{(6)}\right)_{j m}$ in $\mathrm{GeV}^{-2}$ obtained using IceCube data $[1,2]$.

\begin{tabular}{|c|c|c|c|c|}
\hline$d$ & $j$ & Lower bound & Coefficient & Upper bound \\
\hline 4 & 0 & $-4 \times 10^{-19}<$ & $\left(c_{\mathrm{of}}^{(4)}\right)_{00}$ & \\
\hline \multirow[t]{3}{*}{4} & 1 & $-1 \times 10^{-17}<$ & $\left(c_{\mathrm{of}}^{(4)}\right)_{10}$ & $<4 \times 10^{-17}$ \\
\hline & & $-3 \times 10^{-17}<$ & $\operatorname{Re}\left(c_{\text {of }}^{(4)}\right)_{11}$ & $<2 \times 10^{-17}$ \\
\hline & & $-2 \times 10^{-17}<$ & $\operatorname{Im}\left(c_{\text {of }}^{(4)}\right)_{11}$ & $<2 \times 10^{-17}$ \\
\hline \multirow[t]{5}{*}{4} & 2 & $-1 \times 10^{-17}<$ & $\left(c_{\mathrm{of}}^{(4)}\right)_{20}$ & $<7 \times 10^{-17}$ \\
\hline & & $-2 \times 10^{-17}<$ & $\operatorname{Re}\left(c_{\text {of }}^{(4)}\right)_{21}$ & $<3 \times 10^{-17}$ \\
\hline & & $-2 \times 10^{-17}<$ & $\operatorname{Im}\left(c_{\text {of }}^{(4)}\right)_{21}$ & $<5 \times 10^{-17}$ \\
\hline & & $-5 \times 10^{-17}<$ & $\operatorname{Re}\left(c_{\text {of }}^{(4)}\right)_{22}$ & $<2 \times 10^{-17}$ \\
\hline & & $-3 \times 10^{-17}<$ & $\operatorname{Im}\left(c_{\mathrm{of}}^{(4)}\right)_{22}$ & $<4 \times 10^{-17}$ \\
\hline 6 & 0 & $-3 \times 10^{-31}<$ & $\left(c_{\mathrm{of}}^{(6)}\right)_{00}$ & \\
\hline \multirow[t]{3}{*}{6} & 1 & $-2 \times 10^{-28}<$ & $\left(c_{\mathrm{of}}^{(6)}\right)_{10}$ & $<9 \times 10^{-28}$ \\
\hline & & $-6 \times 10^{-28}<$ & $\operatorname{Re}\left(c_{\text {of }}^{(6)}\right)_{11}$ & $<5 \times 10^{-28}$ \\
\hline & & $-3 \times 10^{-28}<$ & $\operatorname{Im}\left(c_{\text {of }}^{(6)}\right)_{11}$ & $<3 \times 10^{-28}$ \\
\hline \multirow[t]{5}{*}{6} & 2 & $-4 \times 10^{-28}<$ & $\left(c_{\mathrm{of}}^{(6)}\right)_{20}$ & $<7 \times 10^{-27}$ \\
\hline & & $-1 \times 10^{-27}<$ & $\operatorname{Re}\left(c_{\text {of }}^{(6)}\right)_{21}$ & $<2 \times 10^{-27}$ \\
\hline & & $-1 \times 10^{-27}<$ & $\operatorname{Im}\left(c_{\text {of }}^{(6)}\right)_{21}$ & $<3 \times 10^{-27}$ \\
\hline & & $-5 \times 10^{-27}<$ & $\operatorname{Re}\left(c_{\text {of }}^{(6)}\right)_{22}$ & $<6 \times 10^{-28}$ \\
\hline & & $-1 \times 10^{-27}<$ & $\operatorname{Im}\left(c_{\mathrm{of}}^{(6)}\right)_{22}$ & $<4 \times 10^{-27}$ \\
\hline \multirow[t]{7}{*}{6} & 3 & $-1 \times 10^{-26}<$ & $\left(c_{\mathrm{of}}^{(6)}\right)_{30}$ & $<4 \times 10^{-27}$ \\
\hline & & $-2 \times 10^{-27}<$ & $\operatorname{Re}\left(c_{\text {of }}^{(6)}\right)_{31}$ & $<1 \times 10^{-26}$ \\
\hline & & $-5 \times 10^{-27}<$ & $\operatorname{Im}\left(c_{\text {of }}^{(6)}\right)_{31}$ & $<3 \times 10^{-27}$ \\
\hline & & $-2 \times 10^{-27}<$ & $\operatorname{Re}\left(c_{\text {of }}^{(6)}\right)_{31}$ & $<1 \times 10^{-26}$ \\
\hline & & $-4 \times 10^{-27}<$ & $\operatorname{Im}\left(c_{\mathrm{of}}^{(6)}\right)_{32}$ & $<6 \times 10^{-27}$ \\
\hline & & $-5 \times 10^{-27}<$ & $\operatorname{Re}\left(c_{\text {of }}^{(6)}\right)_{33}$ & $<6 \times 10^{-27}$ \\
\hline & & $-1 \times 10^{-26}<$ & $\operatorname{Im}\left(c_{\text {of }}^{(6)}\right)_{33}$ & $<7 \times 10^{-28}$ \\
\hline \multirow[t]{9}{*}{6} & 4 & $-5 \times 10^{-27}<$ & $\left(c_{\mathrm{of}}^{(6)}\right)_{40}$ & $<2 \times 10^{-27}$ \\
\hline & & $-1 \times 10^{-27}<$ & $\operatorname{Re}\left(c_{\text {of }}^{(6)}\right)_{41}$ & $<3 \times 10^{-27}$ \\
\hline & & $-1 \times 10^{-27}<$ & $\operatorname{Im}\left(c_{\text {of }}^{(6)}\right)_{41}$ & $<6 \times 10^{-28}$ \\
\hline & & $-1 \times 10^{-27}<$ & $\operatorname{Re}\left(c_{\text {of }}^{(6)}\right)_{42}$ & $<3 \times 10^{-27}$ \\
\hline & & $-2 \times 10^{-27}<$ & $\operatorname{Im}\left(c_{\text {of }}^{(6)}\right)_{42}$ & $<1 \times 10^{-27}$ \\
\hline & & $-1 \times 10^{-27}<$ & $\operatorname{Re}\left(c_{\text {of }}^{(6)}\right)_{43}$ & $<9 \times 10^{-28}$ \\
\hline & & $-2 \times 10^{-27}<$ & $\operatorname{Im}\left(c_{\text {of }}^{(6)}\right)_{43}$ & $<1 \times 10^{-27}$ \\
\hline & & $-2 \times 10^{-27}<$ & $\operatorname{Re}\left(c_{\text {of }}^{(6)}\right)_{44}$ & $<1 \times 10^{-27}$ \\
\hline & & $-5 \times 10^{-28}<$ & $\operatorname{Im}\left(c_{\text {of }}^{(6)}\right)_{44}$ & $<1 \times 10^{-27}$ \\
\hline
\end{tabular}

competitive upper bound on $\dot{c}^{(4)}$ is challenging. An interesting option is time-of-flight measurements, which are sensitive to ${ }^{(4)}$ because the time delay $\Delta t$ in a neutrino pulse of energy $|\boldsymbol{p}|$ arriving from a source at distance $L$ is $\Delta t \approx L|\boldsymbol{p}|^{d-4} \dot{c}^{(d)}[5]$. For $d \geq 6$ this result is typically less sensitive than the constraint (8) from cosmic rays, but for $d=4$ it is independent of energy and offers interesting prospects. For example, time delays of $10 \mathrm{~s}$ or better could be observable in future neutrino-photon coincidence measurements from a gamma-ray burst at a Gpc 
distance, which would yield the constraint $\dot{c}^{(4)} \lesssim 10^{-16}$ for $d=4$ along with the bounds ${ }_{c}^{(d)} \lesssim 10^{8-6 d} \mathrm{GeV}^{4-d}$ for arbitrary $d$ assuming an accompanying emission of highenergy PeV neutrinos. Another option could be neutrino pulse dispersion, which produces a velocity difference $\delta v$ between neutrinos of energies $\left|\boldsymbol{p}_{1}\right|$ and $\left|\boldsymbol{p}_{2}\right|$ given by [5] $\delta v=\mid(d-3)\left(\left|\boldsymbol{p}_{2}\right|^{d-4}-\left|\boldsymbol{p}_{1}\right|^{d-4}\right) \dot{c}^{\circ(d)}$. However, for $d=4$ this vanishes, while for $d \geq 6$ the resulting constraints are weaker than the cosmic-ray bound (8).

In this work, we have demonstrated that observations of high-energy astrophysical neutrinos place stringent limits on deviations from the laws of relativity. The first IceCube events already improve the constraints on Lorentz violation by factors ranging from about a million to about
$10^{20}$, making them competitive with other extreme astrophysical limits from photons and fermions. The sensitivities achieved have surpassed the level at which Planck-suppressed effects could be expected to emerge, and as such they place tight constraints on models involving Planck-suppressed Lorentz violation. Future observations in this new arena of astrophysics offer excellent prospects for further sharpening constraints on the available coefficient space.

This work was supported in part by the Department of Energy under Grant No. DE-FG02-13ER42002 and by the Indiana University Center for Spacetime Symmetries.
[1] M. G. Aartsen et al. (IceCube Collaboration), Phys. Rev. Lett. 111, 021103 (2013).

[2] M. G. Aartsen et al. (IceCube Collaboration), Science 342, 1242856 (2013).

[3] V. A. Kostelecký and S. Samuel, Phys. Rev. D 39, 683 (1989); V. A. Kostelecký and R. Potting, Nucl. Phys. B359, 545 (1991); Phys. Rev. D 51, 3923 (1995).

[4] L. B. Auerbach et al., Phys. Rev. D 72, 076004 (2005); P. Adamson et al., Phys. Rev. Lett. 101, 151601 (2008); Phys. Rev. Lett. 105, 151601 (2010); Phys. Rev. D 85, 031101 (2012); R. Abbasi et al., Phys. Rev. D 82, 112003 (2010); A. A. Aguilar-Arevalo et al., Phys. Lett. B 718, 1303 (2013); T. Katori, Mod. Phys. Lett. A 27, 1230024 (2012); Y. Abe et al., Phys. Rev. D 86, 112009 (2012); B. Rebel and S. Mufson, Astropart. Phys. 48, 78 (2013); J. S. Díaz, T. Katori, J. Spitz, and J. M. Conrad, Phys. Lett. B 727, 412 (2013); V. A. Kostelecký and M. Mewes, Phys. Rev. D 69, 016005 (2004); Phys. Rev. D 70, 076002 (2004); J. S. Díaz, V. A. Kostelecký, and M. Mewes, Phys. Rev. D 80, 076007 (2009).

[5] V. A. Kostelecký and M. Mewes, Phys. Rev. D 85, 096005 (2012).

[6] J. S. Díaz, V. A. Kostelecký, and R. Lehnert, Phys. Rev. D 88, 071902(R) (2013).

[7] D. Colladay and V. A. Kostelecký, Phys. Rev. D 55, 6760 (1997); 58, 116002 (1998); V. A. Kostelecký, Phys. Rev. D 69, 105009 (2004).

[8] V. A. Kostelecký and N. Russell, Rev. Mod. Phys. 83, 11 (2011); arXiv:0801.0287v6.

[9] A. Chodos, A. Hauser, and V. A. Kostelecký, Mod. Phys. Lett. A 07, 467 (1992); V. A. Kostelecký, Topics on Quantum Gravity and Beyond, edited by F. Mansouri and J. J. Scanio (World Scientific,
Singapore, 1993); A. Chodos and V. A. Kostelecký, Phys. Lett. B 336, 295 (1994).

[10] S. Coleman and S. L. Glashow, Phys. Rev. D 59, 116008 (1999).

[11] A. G. Cohen and S. L. Glashow, Phys. Rev. Lett. 107, 181803 (2011).

[12] See, for example, I. Cholis and D. Hooper, J. Cosmol. Astropart. Phys., 06 (2013) 030; M. D. Kistler, T. Stanev, and H. Yuksel, arXiv:1301.1703; O.E. Kalashev, A. Kusenko, and W. Essey, Phys. Rev. Lett. 111, 041103 (2013); H.-N. He, T. Wang, Y.Z. Fan, S. M. Liu, and D. M. Wei, Phys. Rev. D 87, 063011 (2013); N. Gupta, Astropart. Phys. 48, 75 (2013); D. B. Fox, K. Kashiyama, and P. Mészarós, Astrophys. J. 774, 74 (2013); F. W. Stecker, Phys. Rev. D 88, 047301 (2013); K. Murase and K. Ioka, Phys. Rev. Lett. 111, 121102 (2013); R. Laha, J. F. Beacom, B. Dasgupta, S. Horiuchi, and K. Murase, Phys. Rev. D 88, 043009 (2013); L. A. Anchordoqui et al., arXiv:1306.5021; A. Neronov, D. V. Semikoz, and C. Tchernin, arXiv:1307.2158.

[13] E. Borriello, S. Chakraborty, A. Mirizzi, and P. D. Serpico, Phys. Rev. D 87, 116009 (2013); F. W. Stecker, arXiv:1306.6095.

[14] V. A. Kostelecký and M. Mewes, Phys. Rev. D 88, 096006 (2013); Phys. Rev. Lett. 110, 201601 (2013); Phys. Rev. D 80, 015020 (2009).

[15] See, for example, G. B. Dantzig and M. N. Thapa, Linear Programming 1: Introduction (Springer, New York, 1997).

[16] R. Bluhm, V. A. Kostelecký, C. D. Lane, and N. Russell, Phys. Rev. D 68, 125008 (2003); Phys. Rev. Lett. 88, 090801 (2002); V. A. Kostelecký and M. Mewes, Phys. Rev. D 66, 056005 (2002).

[17] B. Altschul, J. Phys. Conf. Ser. 173, 012003 (2009). 